\title{
ESTRUCTURA Y COMPOSICIÓN DE LA VEGETACIÓN RIBEREÑA DE LA BARRANCA DEL RÍO TEMBEMBE, MORELOS, MÉXICO
}

\author{
Fernando CAMACHO-Rico ${ }^{1,3}$, IRMa TRejO² y CONSUELO BONFIL ${ }^{1}$ \\ 'Departamento de Ecología y Recursos Naturales, Facultad de Ciencias, Universidad Nacional \\ Autónoma de México, Circuito Exterior s/n, Ciudad Universitaria, México 04510, D.F., México. \\ ${ }^{2}$ Instituto de Geografía, Universidad Nacional Autónoma de México. \\ ${ }^{3}$ Autor para la correspondencia. Correo-e: tovalito@gmail.com
}

\begin{abstract}
Resumen: Se analizó la estructura y la composición de la vegetación ribereña de la porción media-alta del río Tembembe, Morelos, México. Se registró la altura, el diámetro, la cobertura y la identidad taxonómica de cada individuo $1 \mathrm{~cm} \mathrm{D} \mathrm{AP} \mathrm{en} 23$ unidades de muestreo de $100 \mathrm{~m}^{2}$ cada una. En total se registraron 74 especies y 516 individuos. La densidad promedio fue de 2,148 ind. ha ${ }^{-1}$; el área basal promedio de $88.39 \mathrm{~m}^{2}$ ha $^{-1} \mathrm{y}$ la altura promedio (de individuos con DAP $20 \mathrm{~cm}$ ) de $10.5 \mathrm{~m}$. Con base en análisis de clasificación se distinguieron tres comunidades vegetales cuya distribución está asociada al gradiente altitudinal: la primera (G1) en la parte alta (1,700 - 1,650 m s.n.m.), con especies dominantes de afinidad holártica (como Alnus acumi nata); la segunda (G2) en la parte media (1,640 - 1,340 m s.n.m.), dominada por Trema micrantha; y la tercera (G3) en la porción baja (1,210 - 1,110 m s.n.m.), dominada por especies de afinidad neotropical, como Daphnopsis salicifolia. La diversidad, la densidad y la cobertura no difirieron significativamente entre las tres comunidades, pero el área basal fue mayor en las comunidades alta y baja que en la intermedia. La altura promedio del estrato arbóreo disminuyó con la altitud. El presente estudio contribuye al conocimiento de la vegetación ri b e reña del estado de Morelos y propo rciona info rmación ecológica básica para futuros programas de restauración de la vegetación ribereña del río Tembembe.
\end{abstract}

Palabras clave: composición florística, diversidad florística, estructura de la vegetación, gradiente altitudinal, vegetación ribereña.

\begin{abstract}
Structure and composition of the riparian vegetation of the middle-upper basin of the Tembembe river, in the state of Morelos, was analyzed. Height, diameter at breast height, crown area and taxonomic identity of each individual having a DBH $1 \mathrm{~cm}$ were recorded in 23 quadrats of $100 \mathrm{~m}^{2}$ each. A total of 74 species and 516 individuals were found. Mean density was 2,148 ind. ha ${ }^{-1}$, mean basal area was $88.39 \mathrm{~m}^{2} \mathrm{ha}^{-1}$, and mean height (of individuals with DBH $20 \mathrm{~cm}$ ) w as $10.5 \mathrm{~m}$. Classification analysis allowed the discrimination of three communities whose distribution was associated to the altitudinal gradient: the first (G1) in the upper part (1,700 - 1,650 m a.s.l.) was dominated by species (like Alnus acuminata) having a holartic affinity; the second (G2) at an intermediate altitude (1,640 - 1,340 m a.s.1.) was dominated by Trema micrantha; and the third (G3) in the lower portion (1,210 - 1,110 m a.s.1.) dominated by species (like Daphnopsis salicifolia) having a tropical affinity. Diversity, density and crown cover did not differ among them, but mean basal area was largest in the highest and lowest communities than in the intermediate one. Mean height of the tree layer decreased with altitude. This study contributes to the knowledge of the riparian vegetation in Morelos state and provides basic ecological information for future restoration programs of the riparian vegetation of the Tembembe river.
\end{abstract}

Key words: altitudinal gradient, floristic composition, floristic diversity, riparian vegetation, vegetation structure.

L as riberas de los ríos son sistemas abiertos con propiedades físicas y biológicas propias, a lo largo de los cuales se redistribuye el material orgánico transportado por la corriente del río. Debido a la proximidad y la interacción con los cuerpos de agua, la vegetación ribereña tiene una forma lineal característica y constituye una zona de transición entre los sistemas terrestres y los acuáticos (Wyant y Ellis, 1990; Naiman y Décamps, 1997; Naiman et al., 2000; Rosales et al., 2001; Wa rd et al., 2002). Esta vegetación desempeña diversas funciones ecosistémicas, entre las que destacan: (a) la estabilización del suelo de márgenes y orillas, aumentando su resistencia a la erosión, 
(b) la retención de la escorrentía procedente de la cuenca, (c) la utilización de parte importante de los nutrientes disueltos en ella, evitando la eutrofización de las aguas del cauce, (d) la retención de sedimentos, que redunda en la mayor calidad del agua del cauce, y (e) la mejora del paisaje y su valor recreativo (González del Tánago y García de Jalón, 1998).

Las comunidades vegetales ribereñas proporcionan además hábitat y alimento para la fauna y son consideradas corredores para la dispersión de plantas y animales (Meave et al., 1991). Su composición y estructura condicionan tanto los procesos ecosistémicos como las condiciones biológicas de los cuerpos de agua. En suma, estas comunidades han sido consideradas como un sistema continuo que redistribuye materiales a través del paisaje y en el que se presenta una gran variedad de interacciones terrestres y acuáticas (Brinson et al., 1981; Wyant y Ellis, 1990; Naiman et al., 1993; Naiman y Décamps, 1997; Metzger et al., 1997; Beach y Halpern, 2001; Pinheiro y Ribeiro, 2001; Lyon y Sagers, 2002; Ward et al., 2002).

Se ha propuesto que las comunidades ribereñas jugaron un papel importante en el pasado, ya que su gran heterogeneidad ambiental permitió que funcionaran como refugio para un gran número de especies al modificarse su distribución como resultado de cambios climáticos (Gregory et al., 1991; Meave et al., 1991; Meave y Kellman 1994; Rzedowski, 1994; Naiman et al., 2000). En el mismo sentido, Rzedowski (1994) argumentó que la presencia de componentes propios de regiones holárticas en comunidades ribereñas de climas cálidos es un indicio de condiciones climáticas del pasado.

A pesar de su importancia en el mantenimiento de la biodiversidad regional y en la generación de servicios ambientales, la vegetación ribereña en México ha sido relativamente poco estudiada. Rzedowski (1978) la describe como la vegetación que se desarrolla a lo largo de corrientes de agua permanentes o semipermanentes, generalmente formada por árboles de hoja perenne, decidua o parcialmente decidua, con una altura de 4 a 40 m. Estas comunidades se encuentran entre 0 y 2,800 m s.n.m. y comúnmente su distribución es espaciada e irregular. Lot y Novelo (1990) señalan que en ellas predominan los géneros Populus en las zonas áridas y Alnus en regiones más templadas. Se ha descrito también que los gradientes altitudinales presentes en mu chos ríos están asociados con cambios en las vari ables climáticas y en las características de la vegetación ribereña, incluyendo su diversidad, así como sus propiedades estructurales y funcionales (Vannote et al., 1980; Nilsson et al., 1989; Gregory et al., 1991; Hancock et al., 1996; Lykke y Goudiaby, 1999; Lyon y Sagers, 2002; Nilsson y Svedmark, 2002; Ward et al., 2002).

Este estudio tuvo como objetivo describir la estructura y la composición de la vegetación ribereña del río Tembembe, en el noroeste del estado de Morelos (México), en el tramo altitudinal comprendido entre 1,710 y 1,110 m s.n.m., así como caracterizar los cambios en la composición de la vegetación a lo largo de dicho gradiente. Con ello se pretende contribuir al conocimiento de la vegetación ribereña del estado de Morelos y proporcionar información ecológica básica para los programas de restauración ecológica que se desarrollan en la cuenca del río Tembembe.

\section{Área de estudio}

La región en la que se realizó el presente estudio comprende la porción noroccidental del estado de Morelos; forma parte de la subprovincia denominada Cuenca del Balsas-Mezcala (Fries, 1960). El río Tembembe nace en la sierra del Chichinautzin, a 3,350 m s.n.m (figura 1). La totalidad de la cuenca del río Tembembe tiene una superficie de 32,535 ha y es de tipo exorreica; tiene una longitud axial de $45 \mathrm{~km}$ y un ancho promedio de $16 \mathrm{~km}$ (INEGI, 1999a, b; Gómez, 2003).

Según Gómez (2003), la zona de estudio se localiza en la subcuenca media alta del río Tembembe, que tiene una superficie de 9,062 ha. Esta parte del cauce serpentea entre afloramientos de conglomerados de arenisca, dando origen a una barranca bien definida, que en algunas partes llega a tener más de $50 \mathrm{~m}$ de profundidad (CETENAL, 1979a, b; INEGI 1999a, b). La porción norte de la zona de estudio forma parte de la formación Cuernavaca, que consiste en afloramientos de rocas ígneas intrusivas, extrusivas y sedimentarias, mientras que la porción sur corresponde a la sierra de Xochicalco y está formada por una sucesión de capas de calizas y dolomitas interestratificadas (Fries, 1960). En la parte norte predominan los suelos de tipo Feozem háplico y secundariamente los Vertisoles pélicos de textura media, poco profundos, mientras que al sur, en la sierra de Xochicalco, los suelos son Rendzinas de textura media y fase lítica rocosa, de entre 10 y $50 \mathrm{~cm}$ de profundidad (CETENAL, 1976a, b).

El clima en la parte norte de la zona de estudio es templado subhúmedo (Ahuatenco, 1,950 m s.n.m.), con una temperatura media anual de $17.4^{\circ} \mathrm{C}$ y precipitación anual de 1,160 mm, mientras que en la parte baja (El Rodeo, 1,100 m s.n.m.) es cálido subhúmedo con una temperatura media anual de $22.8^{\circ} \mathrm{C}$ y una precipitación anual de 1,055 $\mathrm{mm}$. La temporada de secas se extiende de noviembre a mayo (figuras 1a, b y c). Asociado a este gradiente altitudinal se presentan cambios en las comunidades vegetales: en los bordes y parte superior del norte de la barranca se encuentra un bosque caducifolio de encino, con Quercus magnoliifolia y $Q$. glaucoides como componentes principales, acompañados de pinos de baja talla. Estas comunidades son sustituidas gradualmente por bosque tropical caducifolio (BTC), particularmente hacia el sur de la zona de estudio, en la sierra de Xochicalco; dominan diversas 

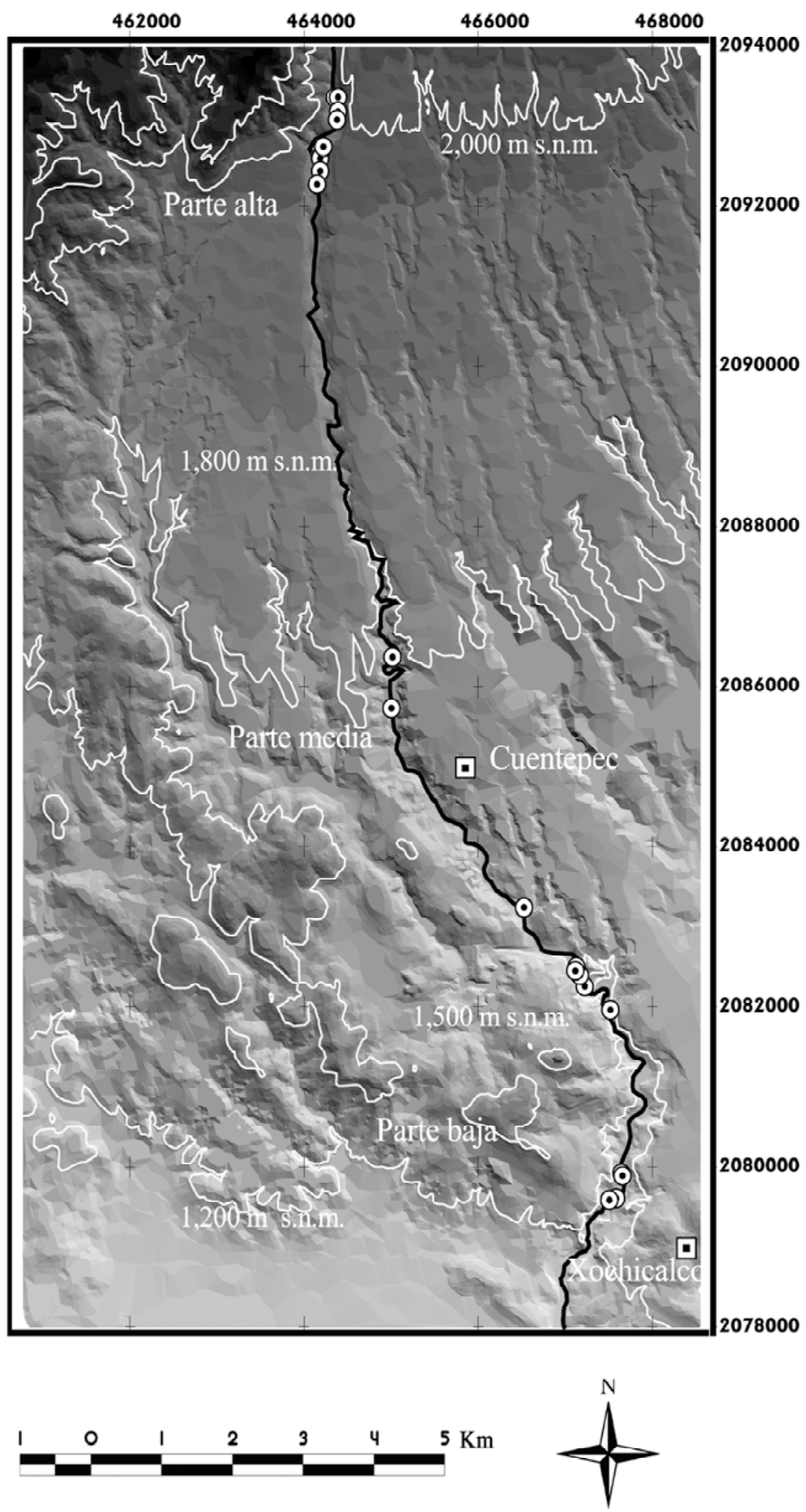
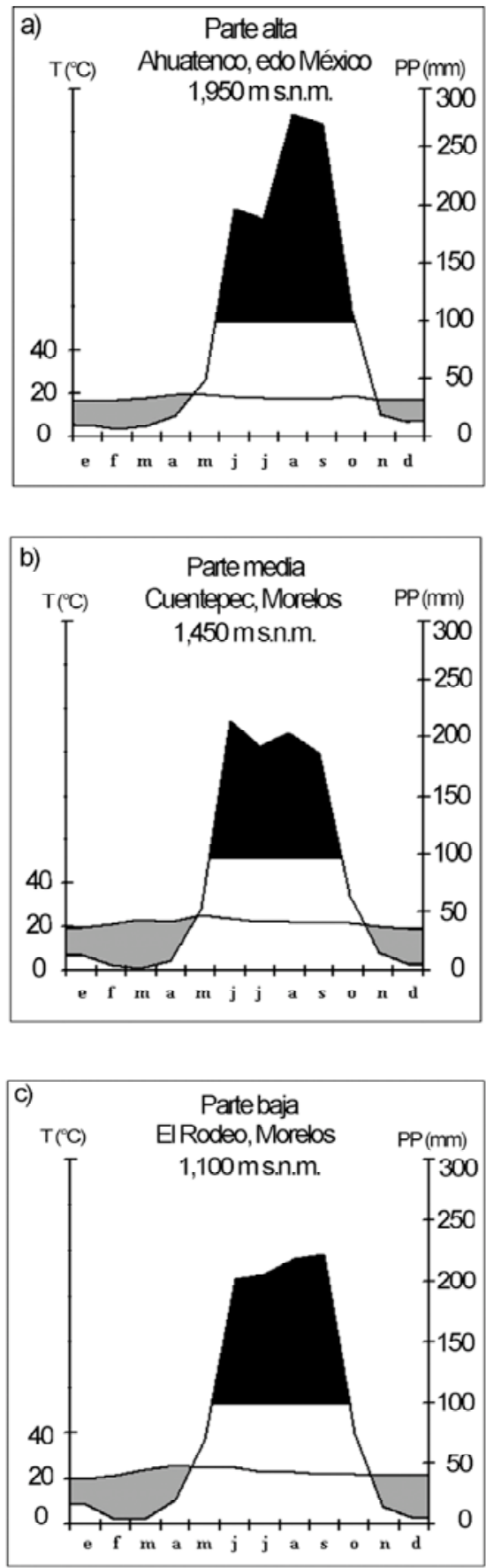

$\square$ Poblaciones humanas

- Unidades de muestreo

- Río Tembembe

Figura 1. Modelo digital del terreno (sistema ITRF92 (Z-14)). Diagramas ombrotérmicos de las estaciones climatológicas de: (a) Ahuatenco, Edo. de México, (b) Cuentepec, Mor., y (c) El Rodeo, Mor. 
especies del género Bursera, además de géneros como Ceiba, Ipomoea, Plumeria, Conzattia, Acacia, Sapium y Lysiloma (CETENAL, 1976c, d; Piña, 2005). En el tramo intermedio se presentan elementos arbóreos del BTC mezclados con manchones de Q. glaucoides.

\section{Materiales y métodos}

Para muestrear la vegetación se usaron unidades de forma rectangular de $20 \times 5 \mathrm{~m}$, iguales a las usadas por LebrijaTrejos (2001). Éstas se colocaron en los parches remanentes de vegetación ribereña que mostraran evidencias de poca perturbación antropogénica. Las unidades se numeraron en orden creciente; la primera se ubicó en el punto más alto del gradiente altitudinal, mientras que la última se estableció en la porción más baja.

En cada una se registró la altura, el diámetro a la altura del pecho (DAP) y los radios mayor y menor de la copa, de todos los individuos con DAP $1 \mathrm{~cm}$. Con estas $\mathrm{v}$ ariables se calculó el valor de importancia relativa (VIR; Curtis y McIntosh, 1951) de cada especie, como la suma de la frecuencia relativa, la densidad relativa y la dominancia relativa (área basal relativa). En el caso de las lianas, se registró el diámetro basal de los individuos enraizados en la unidad de muestreo. Los individuos fueron recolectados para su posterior identificación en gabinete, así como para la formación de un herbario de la zona; además, se tomaron fotografías digitales para la formación de un catálogo de imágenes de las especies registradas. Las coordenadas de cada unidad de muestreo se ubicaron en el sistema ITRF92 (Z-14), y se registró la altitud y la pendiente (perpendicular al cauce del río).

Para analizar la diversidad se usaron los índices de Shannon-Wiener (con logaritmo natural), Inverso de Simpson y el índice de equitatividad de Pielou; para medir la diversidad $\beta$ se calculó el coeficiente de Sørensen $\left(\mathrm{C}_{\mathrm{s}}\right)$. Para agrupar las unidades de muestreo se realizó un análisis de clasificación multivariado aglomerativo, usando el Método de Ward y las distancias euclidianas como medidas de disimilitud de la matriz de datos de presencia-ausencia de especies (STATSOFT INC., 2001). La relación entre el cambio en la composición de especies y el gradiente altitudinal se evaluó mediante un análisis de ordenación (DCA, correspondencias sin tendencia) con el programa PC-ORD para Windows (McCune y Meffor, 1999). Se obtuvieron correlaciones lineales entre las variables registradas para cada unidad de muestreo (i.e. altitud y pendiente) y los ejes de ordenación.

\section{Resultados}

Estructura. Se registraron 516 individuos en 23 unidades de muestreo (U), $56 \%$ de los cuales tuvieron una altura $>4 \mathrm{~m}$, mientras que $38 \%$ tuvieron diámetros $<5 \mathrm{~cm}$. Siete especies de árboles (23\% de los individuos) que presentaron una altura promedio de $9.9 \mathrm{~m}$, aportaron $58.6 \%$ del área basal total. En el cuadro 1 se muestra el número de individuos, el área basal y la cobertura (estandarizadas a una hectárea), así como la altura promedio de los individuos con DAP $20 \mathrm{~cm}$ par a cada unidad de muestreo. Entre las especies con mayor densidad estuvieron Randia aff. canescens (13.7\%), Solanum aligerum (10\%), Daphnopsis salicifolia (10.2\%) y Alnus acuminata (4.8\%); éstas representaron $39 \%$ de la densidad total.

El área basal promedio ( \pm 1 D.E.) fue de $0.70 \pm 0.7 \mathrm{~m}^{2}$ (en $100 \mathrm{~m}^{2}$ ); para esta variable se registró el mayor coeficiente de variación (67\%), ya que los valores oscilaron entre $0.7 \mathrm{~m}^{2}$ (U13) y $1.92 \mathrm{~m}^{2}$ (U3). Al igual que con la densidad, un pequeño grupo de especies aportó la mayor proporción del área basal total (46.6\%): Alnus acuminata (13.4\%), Lysiloma acapulcense (8.9\%), Astianthus vimi nalis (7.6\%) y Daphnopsis salicifolia (6.6\%).

La altura promedio ( \pm 1 D.E.) de los individuos con DAP

$20 \mathrm{~cm}$ fue de $9.8 \pm 4.8 \mathrm{~m}$. El v alor mínimo fue $5.3 \mathrm{~m}$ (U18) y el máximo 14.7 m (U7). Las especies con mayor altura fueron Heliocarpus terebinthaceus, Alnus acumina ta, Fraxinus uhdei, Salix humboldtiana, Trema micrantha, Ficus insipida, Erythrina brevifolia y Diospyros digyna. La cobertura excedió $100 \%$ en todos los casos (cuadro 1); el valor mínimo fue $104 \mathrm{~m}^{2}$ (U2) y el máximo $426 \mathrm{~m}^{2}$ (U7).

Las 10 especies que obtuvieron un mayor VIR fueron, en orden decreciente: Alnus acuminata (22.11), Daphnopsis salicifolia (21.80), Randia aff. canescens (20.25), Lysiloma acapulcense (17.10), Astianthus vimi nalis (14.75), Solanum aligerum (14.53), Salix humbold tiana (12.33), Sapium macrocarpum (11.41) y Critonia quadrangularis (8.09). En el apéndice 1 se presentan los valores de las variables estructurales y el valor de importancia relativa de todas las especies registradas.

Composición florística. Se registraron 74 especies pertenecientes a dos divisiones, Coniferophyta y Magnoliophyta, y dos clases de esta última, Magnoliopsida y Liliopsida. De las familias presentes, la mejor representada fue Leguminosae con ocho géneros y 11 especies (15\%), seguida por Asteraceae con seis géneros y seis especies (8\%), y por Euphorbiaceae con tres géneros y tres especies (4\%). Además de éstas, las familias Solanaceae, Meliaceae y Moraceae estuvieron representadas por tres especies (4\%) aunque en un menor número de géneros (figura 2). Los árboles fueron la forma de crecimiento predominante, con $45.9 \%$ del total de las especies, seguidos por los arbustos con $44.5 \%$ y por último por las lianas, con $9.4 \%$.

Diversidad. El número promedio de especies por unidad fue 8.0. El número más bajo de especies (3) se presentó en la unidad 9 (U9), mientras que en la U15 se encontraron 14 especies, que corresponde a la mayor riqueza encontrada. 
Cuadro 1. Estructura de la vegetación ribereña del río Tembembe, Morelos. Los subrayados corresponden a los valores mínimos y las negritas a los máximos. D.E. = desviación estándar, C.V. = coeficiente de variación.

\begin{tabular}{|c|c|c|c|c|}
\hline Unidad & $\begin{array}{l}\text { Densidad } \\
\text { (ind. ha-1) }\end{array}$ & $\begin{array}{l}\text { Cobertura } \\
(\mathrm{m} 2 \text { ha-1) }\end{array}$ & $\begin{array}{c}\text { Área basal } \\
\text { (m2 ha-1) }\end{array}$ & $\begin{array}{l}\text { Promedio } \\
\text { de altura ind. } \\
\text { DAP } 20 \mathrm{~cm} \\
(\mathrm{~m})\end{array}$ \\
\hline 1 & 2600 & 26100 & 83 & 8.9 \\
\hline 2 & 1000 & 10400 & 44 & 8.0 \\
\hline 3 & 1400 & $\overline{36700}$ & 190 & 12.2 \\
\hline 4 & 1400 & 19600 & 58 & 11.5 \\
\hline 5 & 2300 & 23200 & 110 & 14.3 \\
\hline 6 & 3300 & 35500 & 140 & 12.2 \\
\hline 7 & 3200 & 42600 & 130 & 14.7 \\
\hline 8 & 2400 & 30000 & 114 & 12.4 \\
\hline 9 & 2800 & 33500 & 54 & 12.2 \\
\hline 10 & 4200 & 41200 & 21 & 7.7 \\
\hline 11 & 2000 & 20800 & 15 & 9.0 \\
\hline 12 & 1600 & 15800 & 35 & 6.6 \\
\hline 13 & 1200 & 19200 & 206 & 7.8 \\
\hline 14 & 2400 & 24000 & 67 & 7.0 \\
\hline 15 & 3100 & 26700 & 35 & 5.5 \\
\hline 16 & 2400 & 21200 & $\underline{11}$ & 8.0 \\
\hline 17 & 2600 & 21000 & $\overline{147}$ & 5.5 \\
\hline 18 & 2100 & 26500 & 78 & $\underline{5.3}$ \\
\hline 19 & 1500 & 13900 & 31 & 7.2 \\
\hline 20 & 1700 & 26000 & 148 & 8.6 \\
\hline 21 & 2300 & 24300 & 75 & 6.7 \\
\hline 22 & $\underline{900}$ & 14400 & 63 & 7.4 \\
\hline 23 & 1000 & 25900 & 178 & 10.5 \\
\hline Promedio & 2147.83 & 25152.17 & 88.39 & 9.0 \\
\hline D.E. & 846.85 & 8456.30 & 58.43 & 2.83 \\
\hline C.V. (\%) & 39.43 & 33.62 & 66.10 & 88.03 \\
\hline
\end{tabular}

En el cuadro 2 se muestran los valores de los índices de diversidad de cada unidad de muestreo. El valor promedio del índice de Shannon-Wiener fue de 1.69, mientras que el valor promedio del inverso de Simpson fue de 6.16 y el del índice de equitatividad fue de 0.84 . El mayor coeficiente de variación (50.9\%) se presentó en el inverso de Simpson; los otros dos índices presentaron una variación baja (< $35 \%)$.

El valor máximo del coeficiente de similitud de Sørensen fue de $75 \%$ (U19 y U17), mientras que las unidades 6 y 7 por un lado, y 14 y 18 por el otro, compartieron el mayor número de especies (siete). La distribución de frecuencias del índice de Sørensen muestra que el mayor número de observaciones se presentó en la primera categoría (0 a 6\%), mientras que la categoría con menor número de observaciones fue la de 74 a $79 \%$ (figura 3 ).

Diferencias entre las unidades de muestreo. El dendrograma producido por el análisis de clasificación se muestra en la figura 4. A una distancia relativa de $90 \%$ se distinguen dos grupos, y con una distancia relativa de $55 \%$ como umbral de corte es posible reconocer tres grupos bien definidos. Éstos son: (a) G1, formado por las unidades 1, 2, $3,4,5,6,7$ y 8 ; (b) G2, que comprende las unidades 9,10 , 11,12 y 13; y (c) G3, que incluye las unidades $14,15,16$, $17,18,19,20,21,22$ y 23.

En el diagrama de ordenación producido por el DCA se distinguen dos nubes de puntos a lo largo del eje 1 de ordenación (valor propio [eigenvalue $]=0.908$; varianza acumulada $=11.7 \%$ ). La primera está formada por las unidades

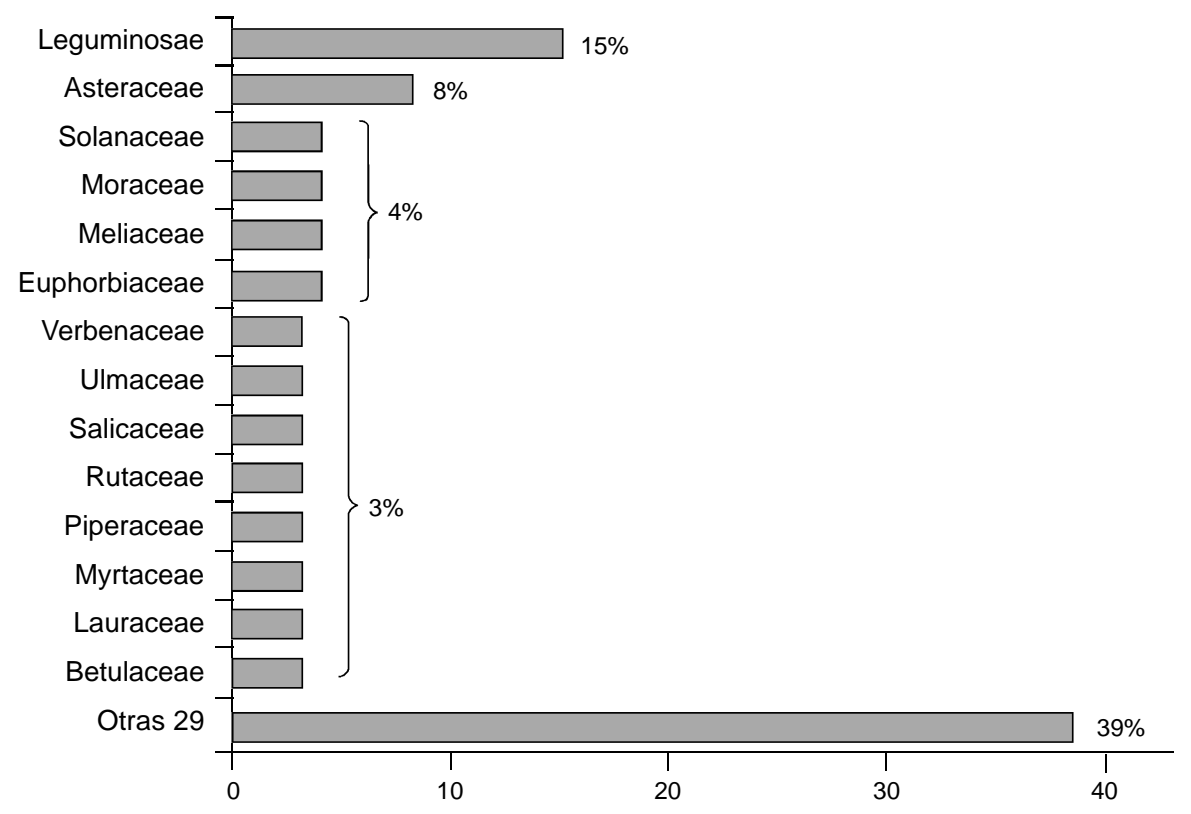

Figura 2. Distribución de frecuencias de las familias de la vegetación ribereña del río Tembembe. 


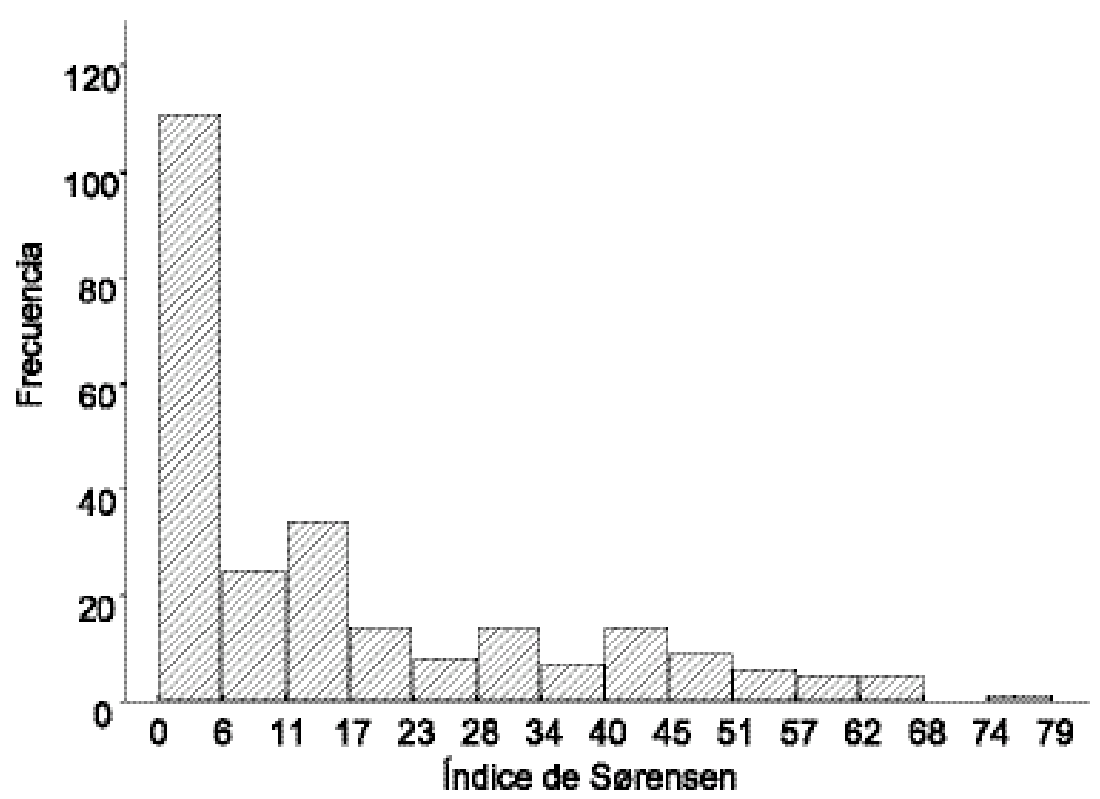

Figura 3. Distribución de frecuencias del Índice de Sørensen.

de la 1 a la 13 (grupos G1 y G2 distinguidos en el análisis de clasificación) y la segunda por las unidades restantes (figura 5). En este análisis Daphnopsis salicifolia y Astianthus viminalis se ubicaron más cercanas en el espacio de ordenación a las unidades de muestreo de menor altitud, mientras que Lysiloma acapulcense se ubicó casi al centro y Randia aff. canescens, Alnus acuminata y Fraxinus uhdei aparecieron cercanas a las unidades de mayor altitud.
Al analizar la relación entre los ejes de ordenación y los valores de las variables ambientales registradas en cada unidad de muestreo, se encontró que la altitud mostró una correlación significativa con el eje 1 de ordenación $\left(R^{2}=\right.$ 0.89, $P=0.0001)$, mientros que la pendiente no mostró correlaciones significativas con ninguno de los dos ejes.

El análisis de las variables estructurales y de diversidad de los tres grupos mostró que la densidad no difirió entre las tres comunidades, aunque el promedio por grupo de la

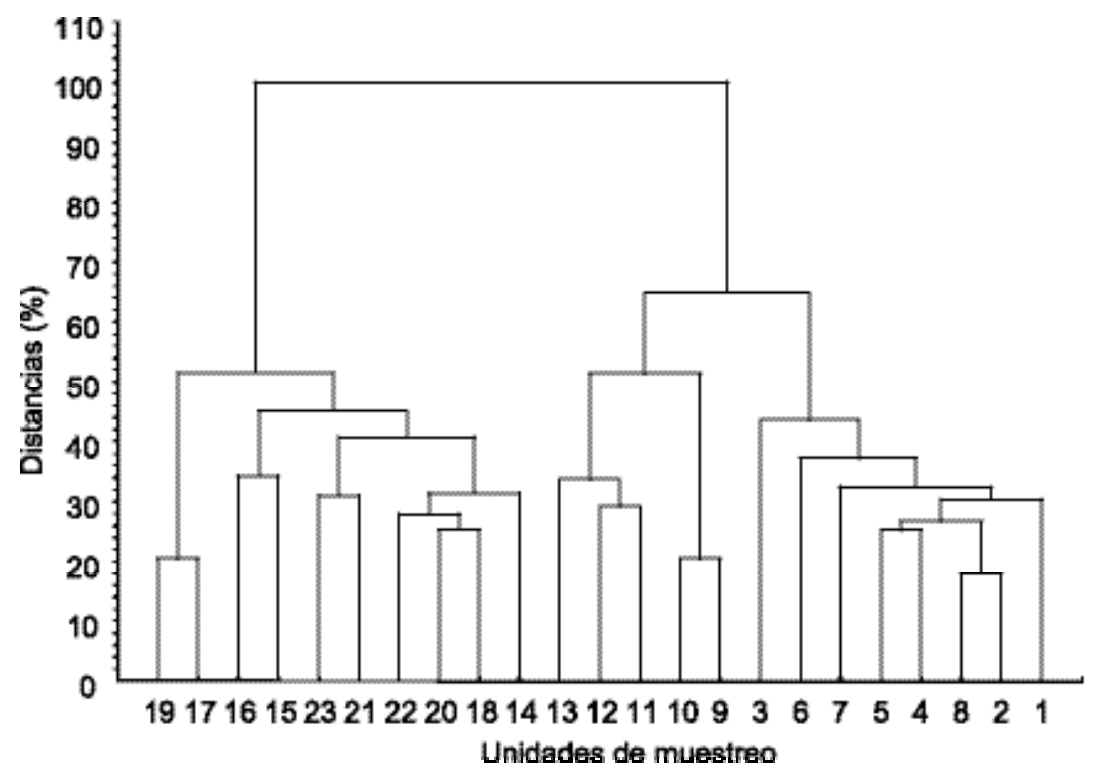

Figura 4. Dendrograma de las unidades de muestreo producido por el método de Ward, usando la matriz de presencia-ausencia y las distancias euclidianas como medida de similitud. 


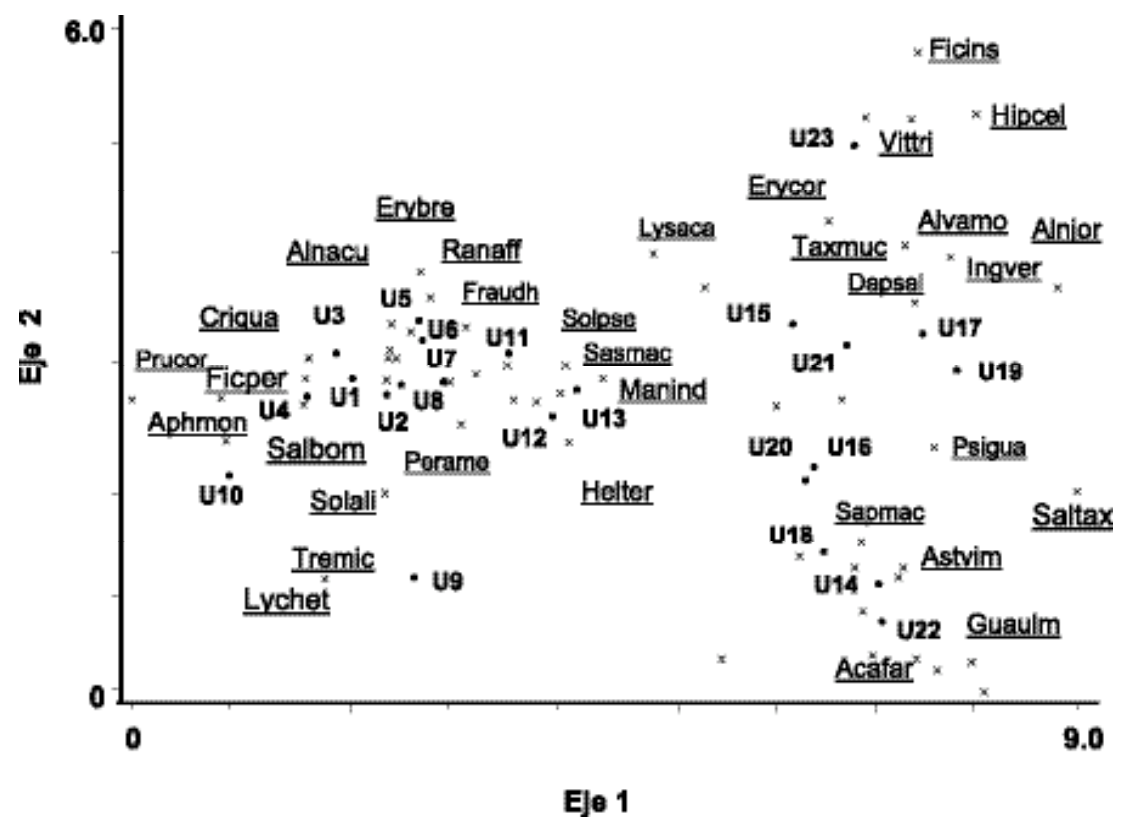

Figu ra5. Diagrama de ordenación de las 23 unidades de mu e s t reo producido por el análisis DECORANA aplicado a la mat riz de presencia-ausencia. Se muestran tanto las unidades (círculos) como las especies más importantes $(\times)$ según su VIR. Valores propios (eingenvalues) del eje 1 y $2,0.908$ y 0.571 , y porcentajes de varianza acumulada, $11.7 \%$ y $19.1 \%$, respectivamente. Los nombres están abreviados con las tres primeras letras del género y de la especie; los nombres completos se pueden consultar en el apéndice 1.

proporción de árboles aumentó a medida que la altitud disminuyó, de tal manera que en la parte alta el cociente de árboles/arbustos fue de 0.78 , en la parte media de 1.49 y en la parte baja de 4.81 (figura 6). La cobertura no difirió significativamente entre las tres comunidades, aunque el área basal promedio sí $(F=5.39 ; P=0.01)$; la mayor fue de 0.94 $\mathrm{m}^{2}$ (en $100 \mathrm{~m}^{2}$ ) y la menor de $0.24 \mathrm{~m}^{2}$, y comespondieron a las comunidades G1 y G2, respectivamente, las cuales difirieron entre sí. Esto se debe a que en la parte alta (G1) el tamaño promedio de un individuo (estimado a partir del área basal promedio por individuo) fue relativamente mayor, y en la intermedia (G2) fue menor (figura 6). La altura (individuos con DAP $20 \mathrm{~cm}$ ) se cor relacionó positivamente con la altitud $\left(R^{2}=0.49 ; P=0.00023\right)$; la diferencia entre la altura promedio del G1 $(11.1 \mathrm{~m})$ y la del G3 $(7.1 \mathrm{~m})$ fue significativa (figura 6). En cuanto a la diversi$\mathrm{dad}$, la riqueza de especies fue similar en las comunidades G1 y G3, pero menor en la G2 (figura 7), aunque los valores de los índices de Shannon-Wiener, Inverso de Simpson y la Equitatividad no difirieron significativamente entre las tres comunidades.

Las especies con los valores de importancia relativa más altos en G1 fueron Alnus acuminata, Randia aff. canescens y Salix humboldtiana (figura 8). En G2 éstos correspondieron a Mangifera indica, Trema micrantha y Solanum aligerum, y en G3 a Daphnopsis salicifolia, Astianthus viminalis y Sapium macrocarpum. Tanto en G1 como en G2 las especies arbustivas Randia aff. canescens (G1) y Solanum aligerum (G2) se encontraron entre los tres primeros
Cuadro 2. Valores de riqueza específica (S), Shannon-Wiener $\left(\mathrm{H}^{\prime}\right)$, inverso de Simpson (1/D) y equitatividad (E) para 23 unidades de muestreo. Los valores en negritas son máximos y los subrayados mínimos. D.E. = desviación estándar, C.V. = coeficiente de variación.

\begin{tabular}{ccccc}
\hline Unidad & $\mathbf{S}$ & $\mathbf{H}^{\mathbf{}}$ & $\mathbf{1 / D}$ & $\mathbf{E}$ \\
\hline 1 & 7 & 1.23 & $\underline{2.32}$ & $\underline{0.63}$ \\
2 & 4 & 1.28 & 4.50 & 0.92 \\
3 & 11 & 1.92 & 4.86 & 0.80 \\
4 & 8 & 1.97 & 11.38 & 0.95 \\
5 & 6 & 1.17 & $\underline{2.32}$ & 0.65 \\
6 & 12 & 2.24 & 8.38 & 0.90 \\
7 & 11 & 2.19 & 9.36 & 0.91 \\
8 & 6 & 1.19 & 2.68 & 0.66 \\
9 & $\underline{3}$ & $\underline{1.08}$ & 3.12 & $\mathbf{0 . 9 8}$ \\
10 & 6 & 1.25 & 2.90 & 0.70 \\
11 & 6 & 1.35 & 3.11 & 0.75 \\
12 & 10 & 2.01 & 7.50 & 0.87 \\
13 & 8 & 1.95 & 9.00 & 0.94 \\
14 & 12 & 2.30 & $\mathbf{1 4 . 5 3}$ & 0.93 \\
15 & $\mathbf{1 4}$ & $\mathbf{2 . 3 3}$ & 11.07 & 0.88 \\
16 & 10 & 1.93 & 6.00 & 0.84 \\
17 & 8 & 1.68 & 4.28 & 0.81 \\
18 & 10 & 1.88 & 5.25 & 0.82 \\
19 & 8 & 1.81 & 6.18 & 0.87 \\
20 & 7 & 1.51 & 3.58 & 0.77 \\
21 & 8 & 1.58 & 3.83 & 0.76 \\
22 & 4 & 1.27 & 4.50 & 0.92 \\
23 & 7 & 1.83 & 11.25 & 0.94 \\
Total & 74 & 3.48 & 19.18 & 0.81 \\
& & & & \\
Promedio & 8 & 1.69 & 6.16 & 0.84 \\
D.E. & 2.81 & 0.40 & 3.48 & 0.10 \\
C.V. $(\%)$ & 34.76 & 23.88 & 50.87 & 12.52 \\
\hline & & & &
\end{tabular}




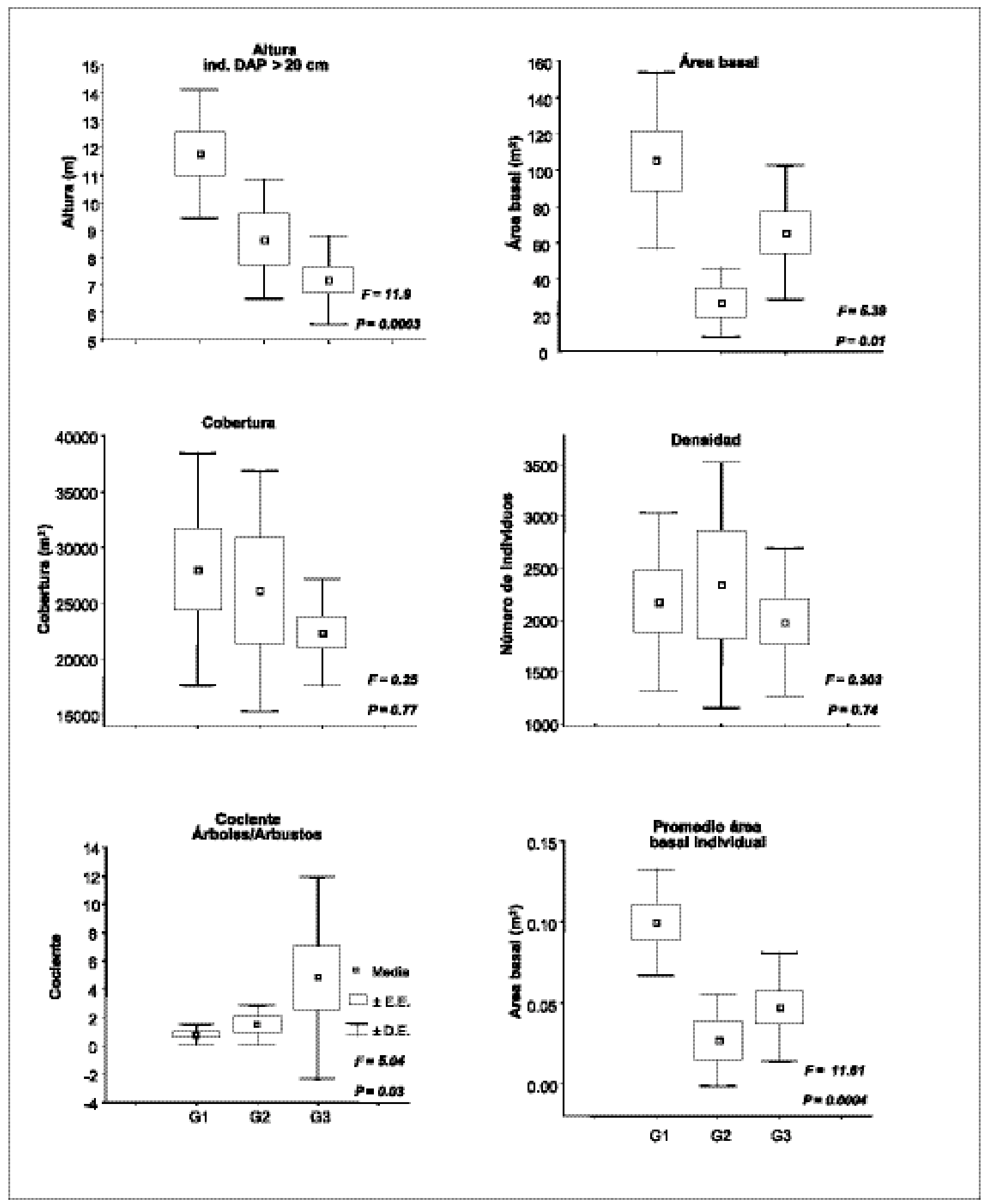

Figura 6. Estructura cuantitativa de los grupos G1, G2 y G3. Se muestra la media, el error estándar (E.E.) y la desviación estándar (D.E.). El estadístico $F$ corresponde al valor del análisis de varianza de una vía. 


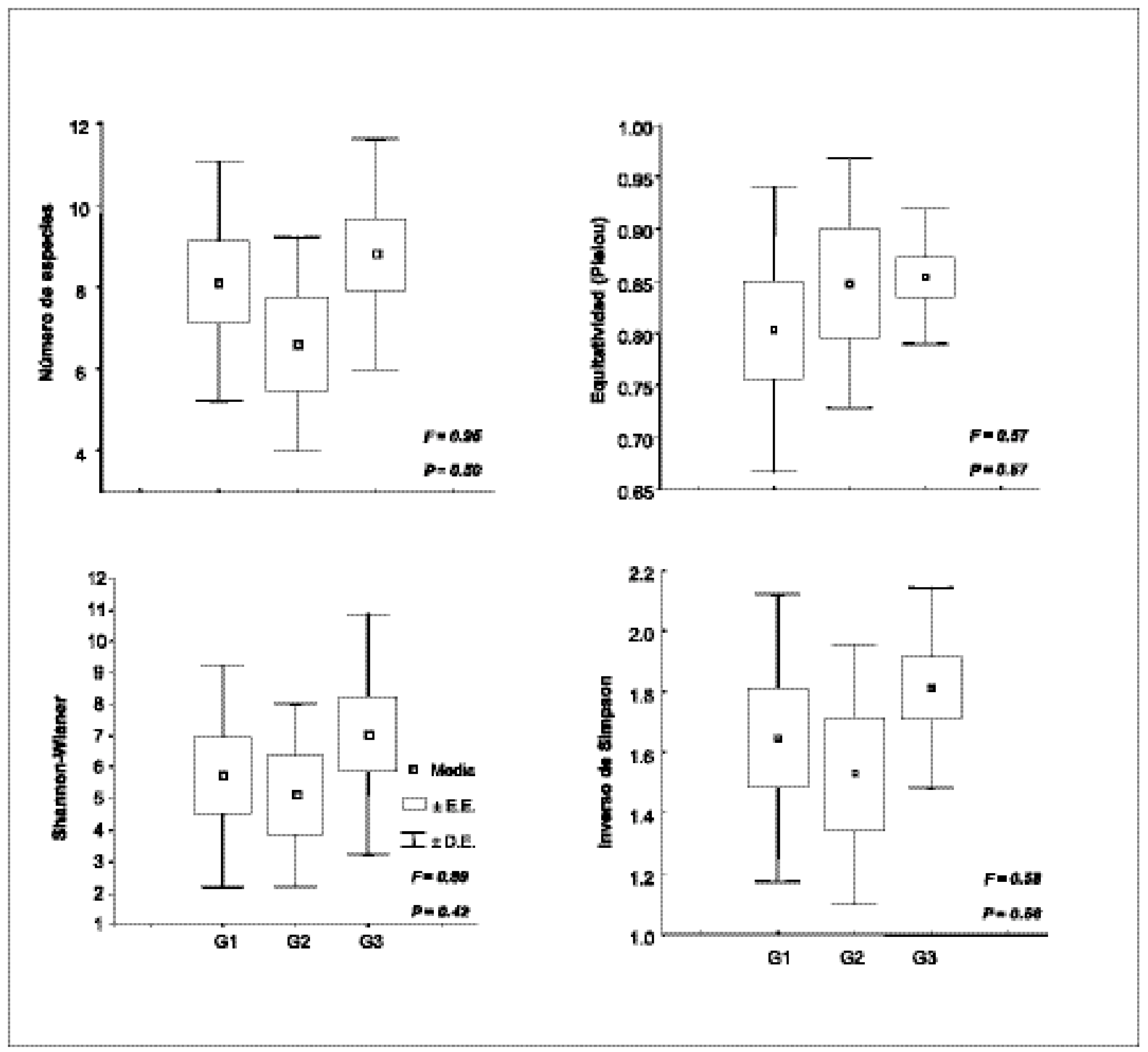

Figura 7. Valores de los índices de diversidad de los grupos G1, G2 y G3. Se muestra la media, el error estándar (E.E.) y la desviación estándar (D.E.). El estadístico $F$ corresponde al valor del análisis de varianza de una vía.

lugares del valor de importancia relativa, debido sobre todo a su alta densidad. Lysiloma acapulcense apareció entre los diez primeros valores de importancia relativa en los tres grupos y alcanzó su valor más alto en G1, mientras que Sapium macrocarpum se encontró entre los diez primeros lugares en los grupos G2 y G3, y alcanzó su valor más alto en este último.

\section{Discusión}

El área muestreada en el presente estudio representa un porcentaje pequeño $(0.23 \mathrm{ha})$ de la ribera del tramo del río analizado. Las grandes discontinuidades en la localización de las unidades de muestreo (figura 1) se explican por el hecho de que en algunas partes se forman cañones estre- chos en los que no existe vegetación, mientras que en áreas extensas ésta ha sido eliminada para establecer huertos y parcelas de cultivo. Por lo tanto, no fue posible realizar un muestreo continuo que permitiera detectar los posibles cambios graduales en la composición de especies presentes.

La comparación de la diversidad de especies encontrada en este estudio con la de otras comunidades del mismo tipo en México muestra que la riqueza específica (74 especies) fue menor que la reportada en los estudios de LebrijaTrejos (2001) en Nizanda (109 especies con DAP $1 \mathrm{~cm}$ en 26 unidades de $100 \mathrm{~m}^{2}$ ) y de Lott et al. (1987) en los arroyos de Chamela (105 especies con DAP $2.5 \mathrm{~cm}$ en 10 unidades de $100 \mathrm{~m}^{2}$ ). Esto puede deberse, como se mencionó, a que la vegetación del Tembembe es muy discontinua 
(a)

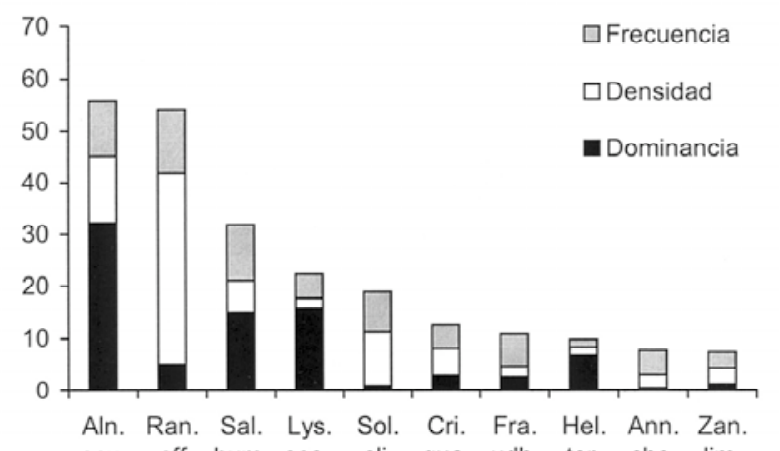

acu. aff hum. aca. ali. qua. udh. ter. che. lim.
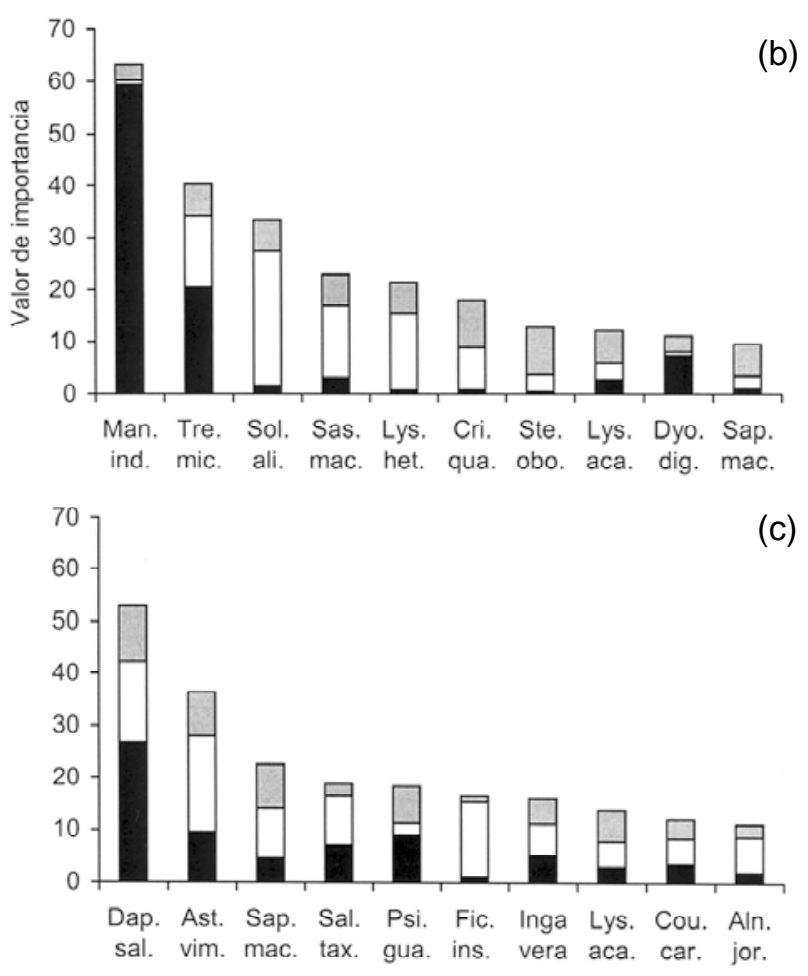

Figura 8. Valores de importancia de Curtis y McIntosh (VIR) de las diez primeras especies de los grupos G1 (a), G2 (b) y G3 (c). Los nombres de cada especie están abreviados y se pueden consultar en el apéndice 1.

y presenta un menor grado de conservación que la de dichas localidades, pero también pudo influir el que la diversidad de especies arbóreas en zonas tropicales suele ser mayor que la registrada en zonas templadas (Rzedowski, 1978; Gentry, 1982; 1995; Challenger 1998; Willig et al., 2003), y la zona de estudio se ubica en una zona de transición entre comunidades templadas y tropicales. Por otro lado, la comparación con la vegetación de los arroyos de Chamela debe tomarse con ciertas reservas, ya que en dichos arroyos las corrientes son intermitentes y las condiciones topográficas favorecen que los componentes ribereños se mezclen con especies propias del bosque tropical subcaducifolio, lo que dificulta su delimitación y caracterización.

En relación con la composición de especies, las familias mejor representadas (Leguminosae, Asteraceae y Euphorbiaceae; figura 2) coinciden con las reportadas por Lebrija-Trejos (2001), quien encontró una alta proporción de leguminosas, seguidas por euforbiáceas y asteráceas, mientras que en la vegetación ribereña de Chamela las familias Leguminosae y Bignoniaceae fueron las más diversas (Lott et al., 1987). La familia Leguminosae es consistentemente la mejor representada debido a que es una de las más diversas en el mundo, y a que está ampliamente distribuida en las regiones tropicales (Gómez-Pompa, 1966; Lavin et al., 2005). La familia Asteraceae es también muy abundante en las regiones tropicales de América. Ambas se establecen en zonas con disturbios recurrentes y su alta importancia (en términos del porcentaje de especies que representan) puede estar relacionada con los disturbios naturales que se presentan en los ecosistemas ribereños debido a las crecidas del río (Mitsch y Gosselink, 1986; Hughes, 1990; Wyant y Ellis, 1990; Gregory et al., 1991; Lenssen et al., 2000; Rosales et al., 2001; Lyon y Sagers, 2002; Nilsson y Svedmark, 2002; Ward et al., 2002).

El recambio de especies puede considerarse alto debido a que la proporción de pares de unidades con valores relativamente bajos del coeficiente de Sørensen fue alta (ver figura 3). Aunque estos resultados pueden estar influidos por las discontinuidades en la vegetación analizada, también puede deberse a que frecuentemente las comunidades ribereñas albergan una alta proporción de la diversidad regional. Por ejemplo, Felfili et al. (2001) reportaron que $33 \%$ de las especies registradas en la región del Cerrado (Brasil) se encuentran en comunidades que sufren inundación, mientras que Lykke y Goudiaby (1999) encontraron que $42 \%$ de las especies arbóreas del parque nacional Delta du Saloum (Senegal) se encuentran en el bosque ribereño. En México, Lebrija-Trejos (2001) encontró que $32 \%$ del total de especies de la región de Nizanda (alrededor de 900; Pérez-García et al., 2005) se encuentran en estas comunidades. En este caso, hasta el momento se han registrado alrededor de 200 especies leñosas en la cuenca alta-media del río Tembembe (tomando en cuenta las registradas por Piña [2005] y las que aquí se reportan), de las cuales alrededor de $37 \%$ forman parte de la vegetación ribereña, valor que es similar a los anteriores.

Los resultados obtenidos apuntan a que en el tramo estudiado del río Tembembe no se establece una sola comunidad ribereña, sino que en ella se pueden distinguir tres comunidades que difieren en composición, afinidad florística y algunas características estructurales, y cuya distribución se relaciona claramente con las variables ambientales 
asociadas al gradiente altitudinal. La comunidad G1 tuvo una marcada afinidad templada, ya que Alnus acuminata es una especie que tolera temperaturas inferiores a $0^{\circ} \mathrm{C}$ y Salix humboldtiana se desarrolla en climas templados; ambas se presentan en las riberas de ríos, canales o arroyos y la primera se considera una especie pionera (Rzedowski, 1978, 1994; Barbour y Billings, 2000; Fralish y Franklin, 2002). Fraxinus uhdei también es abundante en comunidades de clima templado, como encinares húmedos y bosques mesófilos, además de ser característica de la vegetación ribereña (Vázquez-Yanes et al., 1999).

En la comunidad G2 la presencia de Mangifera indica, especie originaria de Asia, se explica porque es ampliamente cultivada en México por sus frutos comestibles (Rzedowski y Rzedowski, 1999); además, se encuentra en el tramo del río con mayor accesibilidad y cercanía al poblado de Cuentepec. Otros elementos importantes fueron Trema micrantha y Sassafridium macrophyllum. La primera es una especie de vegetación secundaria de amplia distribución en el país, tanto en regiones templadohúmedas como cálido-secas, de hábitos ribereños y ruderales (Pennington y Sarukhán, 1998; Vázquez-Yanes et al., 1999), mientras que de la segunda existe muy poca información; cuenta con pocos registros en el Herbario Nacional (MEXU) y fue recolectada en las barrancas de Cuernavaca por Conzatti a finales del siglo XIX y por Miranda en la década de 1950.

En la comunidad G3 la especie con mayor valor de importancia relativa fue Daphnopsis salicifolia, miembro del único género de la familia Thymelaeaceae en México, que posee una fuerte afinidad tropical y es común en el BTC (Standley y Steyermark, 1946; Sosa y Gómez-Pompa, 1994). Astianthus viminalis es una freatofita característica de ríos en áreas de BTC y endémica de México y zonas adyacentes (Rzedowski, 1978; Rzedowski, 1994). Psidium guajava presenta una amplia distribución tropical y es común en sitios perturbados y en suelos con drenaje deficiente (Pennington y Sarukhán, 1998; Vázquez-Yanes et al., 1999); su presencia en la ribera del Tembembe probablemente también ha sido favorecida por sus frutos comestibles. Es notable también la presencia de individuos del género Alnus en zonas relativamente bajas (G3). Sólo algunos elementos, como Lysiloma acapulcense y en menor medida Sapium macrocarpum, se encontraron a lo largo de todo el gradiente altitudinal.

En relación con la comunidad G2, el análisis de clasificación la reconoció como una comunidad con carácter propio, por la dominancia de especies como Mangifera indica, Trema micrantha, Persea americana, Sassafridium macro phyllum y Critonia quadrangularis, que no comparte con las comunidades G1 y G3. Sin embargo, no puede descartarse su posible carácter transicional entre dichas comunidades, ya que el análisis de ordenación no la separa claramente y la ubica relativamente más cerca a G1 (figura 5).
Los datos disponibles no permiten una conclusión definitiva debido a varias razones. La primera es su cercanía al poblado de Cuentepec, lo que probablemente ha causado cambios significativos en la composición y estructura de esta comunidad a lo largo del tiempo, lo que se evidencia por la dominancia de especies cultivadas, como el mango y el aguacate, y de especies propias de ambientes perturbados. Además, presenta valores menores de los índices de diversidad y de algunas variables estructurales (como el área basal; figuras 6 y 7), lo que puede indicar también una mayor perturbación antropogénica. Por otro lado, lascondiciones del río no permiten el establecimiento de vegetación en amplios tramos entre G1 y G3 debido a que el cañón se estrecha y no presenta llanuras de inundación.

Los índices de diversidad de las tres comunidades no presentaron grandes diferencias, en parte porque la riqueza específica fue similar y también porque en cada una se registró una alta densidad de individuos de una o dos especies (que no se comparten entre comunidades) — Randia aff. canescens en la parte alta, Solanum aligerum en la parte media y Daphnopsis salicifolia en la parte baja-, lo que contribuye a homogenizar los valores de los índices.

En relación con la estructura de las tres comunidades, aunque la densidad promedio no difirió entre las comunidades G1 y G3, en la primera se registró una alta densidad de arbustos, que fueron relativamente menos abundantes en la zona más baja (figura 6). Los árboles de la comunidad G1 fueron relativamente más grandes (área basal promedio por individuo mayor en G1 que en G3), mientras que en G3 fueron más abundantes, con un tamaño promedio por individuo menor. En diversos estudios (que no incluyen comunidades ribereñas) se ha documentado que el área basal en bosques templados suele ser mayor que en bosques tropicales secos. Por ejemplo, Gentry (Phillips y Miller, 2002) en un bosque templado (Las Joyas, Manantlán, Jalisco) reportó un área basal promedio de 100 $\mathrm{m}^{2}$ ha $^{-1}$, mientras que Trejo (1998) encontró un área basal promedio de $34.7 \mathrm{~m}^{2} \mathrm{ha}^{-1}$ en un sitio de bosque tropical caducifolio del estado de Morelos, y Gallardo-Cruz et al. (2005) registraron un área basal de $53.2 \mathrm{~m}^{2} \mathrm{ha}^{-1}$ en un bosque tropical caducifolio en la región de Nizanda, Oaxaca. Debido a que no hay suficientes estudios de comunidades ribereñas de México, no se puede establecer si la misma tendencia se registra en este tipo de comunidades.

Los resultados del presente estudio representan una aportación al conocimiento de las comunidades ribereñas de México y pueden ser una contribución para futuros estudios de la vegetación de las numerosas barrancas de la denominada formación Glacis de Buenavista, ya que es probable que compartan características como la composición florística, la estructura y el efecto de las actividades antropogénicas en ellas. Por otro lado, pueden servir de base para el diseño de un programa de restauración de la vegetación ribereña de la porción alta y media del río 
Tembembe, ya que brindan información referente a las especies nativas dominantes y su distribución espacial a lo largo del gradiente altitudinal, y permiten distinguir a las comunidades con un relativo buen estado de conservación de aquéllas que presentan un fuerte impacto antropogénico.

\section{Agradecimientos}

El presente trabajo fue financiado por el proyecto PAPIIT IN-231802 de la Dirección General de Asuntos del Personal Académico de la Universidad Nacional Autónoma de México, del cual obtuvo una beca el primer autor. Agradecemos a Raúl García Barrios del Centro Regional de Investigaciones Multidisciplinarias (UNAM) por su apoyo logístico y académico, y al editor y a dos revisores anónimos por sus sugerencias, que mejora ron el manuscrito original. Ramiro Cruz Durán, Martha Martínez-Gordillo, Jerzy Rzedowski y Rafael Torres apoyaron en la identificación de los ejemplares de herbario. Esteban Benítez, Isabel Cajero, Pedro E. Mendoza, Evelyn Piña, Ulises Ruiz y Juan Ulloa colaboraron en el trabajo de campo.

\section{Literatura citada}

Barbour M.G. y Billings W.D. 2000. North American Terrestrial Vegetation. 2a ed. Cambridge University Press, Cambridge.

Beach E. y Halpern C. 2001. Control of conifer regeneration in managed riparian forests: effects of seed source, substrate, and vegetation. Canadian Journal of Forest Research 31:471-483.

Brinson M.M., Swift B.L., Plantico R.C. y Barclay J.S. 1981. Riparian Ecosystems: Their Ecology and Status. United States Fish and Wildlife Service FWS/OBS-81/17, Washington, D.C.

CETENAL [Comisión de Estudios del Te rrit orio Nacional]. 1976a. Carta Edafológica 1:50,000. Cuern avaca. México, D. F.

CETENAL. 1976b. Carta Edafológica 1:50,000. Tenancingo. México, D.F.

CETENAL. 1976c. Carta de Uso de Suelo y Vegetación 1:50,000. Cuernavaca. México, D.F.

CETENAL. 1976d. Carta de Uso de Suelo y Vegetación 1:50,000. Tenancingo. México, D.F.

CETENAL. 1979a. Carta Geológica 1:50,000. Cuernavaca. México, D.F.

CETENAL. 1979b. Carta Geológica 1:50,000. Tenancingo. México, D.F.

Challenger A. 1998. Utilización y Conservación de los Ecosistemas Terrestres de México. Pasado, Presente y Futuro. Comisión Nacional para el Conocimiento y Uso de la Biodiversidad, Instituto de Biología, UNAM y Agrupación Sierra Madre, S.C., México, D.F.

Curtis J. y McIntosh R. 1951. An upland forest continuum in the praires forest border region of Wisconsin. Ecology 32:476496.

Felfili J., Cunha de Mendoça R., Machado B., Silva da C., Goreth M., William C., Cássio-Sevilha A. y Silva M. 2001. Flora fanerogâmica das matas de galeria e filiares do Brasil central. En: Ribeiro J., Lazarini C. y Sousa-Silva J. Eds. Cerrado: Caracterização e Recuperação de Matas de Galeria, pp. 195-
209, EMBRAPA, Brasilia.

Fralish J. y Franklin B.S. 2002. Taxonomy and Ecology of Woody Plants in North American Forests. John Wiley \& Sons, Nueva York.

Fries C. 1960. Geología del estado de Morelos y partes adyacentes de México y Guerrero, región central meridional de México. Boletín del Instituto de Geología 60:235-260.

Gallardo-Cnz J.A., Meave J.A. y Pérez-García E.A. 2005. Estructura, composición y diversidad de la selva baja caducifolia del Cerro Verde, Nizanda (Oaxaca), México. Boletín de la Sociedad Botánica de México 76:19-35.

Gentry A.H. 1982. Patterns of neotropical plant species diversity. Evolutionary Biology 15:1-84.

Gentry A.H. 1995. Patterns of diversity and floristic composition in Neotropical montane forests. En: Churchill S.P., Balslev H., Forero E. y Luteyn, J.L. Eds. Biodiversity and Conservation of Neotropical Montaine Forests, pp. 103-126. New York Botanical Garden, Nueva York.

Gómez A. 2003. Caracterización del medio físico de la cuenca del río Tembembe empleando sistemas de información geográfica (SIG cuencas). Consultado el 18 de mayo de 2004 en: <http://selper.uabc.mx/Publicacio/Cong11/extenso42.doc.>

Gómez-Pompa A. 1966. Estudios Botánicos en la Región de Misantla, Veracruz. Instituto Mexicano de Recursos Naturales Renovables, A.C., México, D.F.

González del Tánago M. y García de Jalón D. 1998. Restauración de Ríos y Riberas. Fundación Conde del Valle de Salazar y Ediciones Mundi-Prensa, Madrid.

Gregory V.S., Swanson J.F., McKee A.W. y Cummins W.K. 1991. An ecosystem perspective of riparian zones. BioScience 41:540-551.

Hancock C., Laad P. y Froend R. 1996. Biodiversity and management of riparian vegetation in Western Australia. Forest Ecology and Management 85:239-250.

Hughes F. 1990. The influence of flooding regimes on forest distribution and composition in the Tana river floodplain, Kenya. Journal of Applied Ecology 27:475-491.

INEGI [Instituto Nacional de Geografía Estadística e Informática]. 1999a. Carta Topográfica 1:50,000. Cuernavaca. Aguascalientes.

INEGI. 1999b. Carta Topográfica 1:50,000. Tenancingo. Aguascalientes.

Lavin M., Herendeen P. y Wojciechowski M. 2005. Evolutionary rates analysis of leguminosae implicates a rapid diversification of lineages during the Tertiary. Systematic Biology 54:575594.

Lebrija-Trejos E. 2001. Análisis estructural de la vegetación ribereña de Nizanda, Oaxaca México. Tesis de Licenciatura (Biología), Facultad de Ciencias, Universidad Nacional Autónoma de México, México, D.F., 133 pp.

Lenssen J., Menting F., Van der Putten W. y Blom C. 2000. Variation in species composition and species richness within Phragmites australis dominated riparian zones. Plant Ecology 147:137-146.

Lot-Helgueras A. y Novelo A. 1990. Forested wetlands of Mexico. En: Lugo A.E., B rown S. y Brinson M. Eds. Ecosystems of the World: Forested Wetlands, Vol. 15, pp 287298, Elsevier, Amsterdam.

Lott E.J., Bullock S.H. y Solís-Magallanes J.A. 1987. Floristic diversity and structure of upland and arroyo forests of coastal 
Jalisco. Biotropica 19:228-235.

Lykke A. y Goudiaby A. 1999. Structure, floristic composition, and conservation potentials of a remnant gallery forest at "Mare du Dragon", Senegal. Nordic Journal of Botany 19:561573.

Lyon J. y Sagers C. 2002. Correspondence analysis of functional groups in a riparian landscape. Plant Ecology 164:171-183.

McCune M. y Meffor M. 1999. Multivariate analysis of ecological data. Version 4-20. MjM Software, Gleneden Beach, Oregon.

Meave J. y Kellman M. 1994. Maintenance of rainforest diversity in riparian forest of tropical savannas: implications for species conservation during Pleistocene drought. Journal of Biogeography 21:121-135.

Meave J., Kellman M., MacDougall A. y Rosales J. 1991. Riparian habitats as tropical forest refugia. Global Ecology and Biogeography Letters 1:69-76.

Metzger J., Barnacci L. y Goldenberg R. 1997. Pattern of tree species diversity in riparian forest fragments of different widths (SE Brazil). Plant Ecology 133:135-152.

Mitsch W.J. y Gosselink J.G. 1986. Wetlands. Van Nostrand Reinhold, Nueva York.

Naiman R.J., Bilby R., y Bisson P. 2000. Riparian ecology and management in the Pacific coastal rain forest. BioScience 50:996-1011.

Naiman R.J. y Décamps H. 1997. The ecology of interfaces: riparian zones. Annual Review of Ecology and Systematics 28:621658.

Naiman R.J., Décamps H. y Pollock M. 1993. The role of riparian corridors in maintaining regional biodiversity. Ecological Applications 3:209-212.

Nilsson C., Grelsson G., Johansson M. y Sperens U. 1989. Patterns of plant species richness along riverbanks. Ecology 70:77-84.

Nilsson C. y Svedmark M. 2002. Basic principles and ecological consequences of changing water regimes: riparian plant communities. Environmental Management 30:468-480.

Pennington T.D. y Sarukhán J. 1998. Árboles Tropicales de México. Manual para la Identificación de las Principales Especies. Universidad Nacional Autónoma de México y Fondo de Cultura Económica, México, D.F.

Pérez-García E., Meave J.A. y Gallardo-Cruz J.A. 2005. Diversidad beta y diferenciación florística en un paisaje complejo del trópico estacionalmente seco del sur de México. En: Halffter G., Soberón J., Koleff P. y Melic A. Eds. Sobre Diversidad Biológica: El Significado de las Diversidades Alpha, Beta y Gamma, pp. 123-142, Sociedad Entomológica Aragonesa, CONABIO, Grupo Diversitas, Comisión Nacional de Ciencia y Tecnología, Zaragoza.
Phillips O.L. y Miller J. 2002. Global Patterns of Plant Diversity: Alwyn H. Gentry's Forest Transect Data Set. Missouri Botanical Garden, St. Louis, Missouri.

Pinheiro F. y Ribeiro J. 2001. Síndromes de dispersão de sementes em matas de galeria do Distrito Federal. En: Ribeiro J., Lazarini C. y Sousa-Silva J. Eds. Cerrado: Caracterização e Recuperação de Matas de Galeria. 897 pp., EMBRAPA, Brasilia.

Piña E. 2005. Análisis de la estructura y la composición de la selva baja caducifolia con diferentes grados de conservación en la zona de Xochicalco, Morelos, México. Tesis de Licenciatura (Biología) Facultad de Ciencias, Universidad Nacional Autónoma de México, México, D.F., 67 pp.

Rosales J., Petts G. y Knab-Vispo C. 2001. Ecological gradients within the riparian forest of the lower Caura River, Venezuela. Plant Ecology 152:101-118.

Rzedowski J., 1978. Vegetación de México. Limusa, México, D.F.

Rzedowski J., 1994. Geographical affinities of the riparian trees of México. Mémoires de la Société de Biogéographie IV:3744.

Rzedowski J. y Rzedowski G. 1999. Flora del Bajio y Regiones Adyacentes. Fascículo 78. Instituto de Ecología, A.C., Pátzcuaro, Michoacán.

Sosa V. y Gómez-Pompa A. Comp. 1994. Flora de Veracruz. Lista Florística. Instituto de Ecología, A.C., Xalapa, Veracruz.

Standley P.C. y Steyermark J. 1946. Flora of Guatemala, Fieldiana. Botany, Vol. 24, Part IV.

STATSOFT INC. 2001. STATISTICA for Windows. Ver. 6.0, en: <http://www.statsoft.com>

Trejo-Vázquez I. 1998. Distribución y diversidad de selvas bajas de México: relaciones con el clima y el suelo. Tesis de Doctorado (Biología), Facultad de Ciencias, Universidad Nacional Autónoma de México, México, D.F., 210 pp.

Vannote R., Minshall G., Cummins K., Sedell R. y Cushing C. 1980. The river continuum concept. Canadian Journal of Fishing and Aquatic Science 37:130-137.

Vázquez-Yanes C., Batis M.A., Alcocer S.M., Gual-Díaz M. y Dirzo S.C. 1999. Árboles y arbustos potencialmente valiosos para la restauración ecológica y la reforestación. Reporte técnico inédito del proyecto J084. CONABIO - Instituto de Ecología, Universidad Nacional Autónoma de México.

Ward J., Tockner K., Arscott D. y Claret C. 2002. Riverine landscape diversity. Freshwater Biology 47:517-539.

Willig M., Kaufman D. y Stevens R. 2003. Latitudinal gradients of biodiversity: pattern, process, scale, and synthesis. Annual Review of Ecology and Systematics 34:273-309.

Wyant J.G. y Ellis E.J. 1990. Compositional patterns of riparian woodlands in the Rift Valley of northern Kenya. Vegetatio 89:23-37. 
Fernando Camacho-Rico, Irma Trejo y Consuelo Bonfil

Apéndice 1. Variables estructurales de las especies encontradas en el muestreo de la vegetación ribereña de la barranca del río Tembembe, Morelos, México. DR = densidad relativa, FR = frecuencia relativa, $A B R=$ área basal relativa, $V I R=$ valor de importancia relativa.

\begin{tabular}{|c|c|c|c|c|c|c|c|c|}
\hline Especie & $\begin{array}{l}\text { Densidad } \\
\text { (ind. } \text { ha }^{-1} \text { ) }\end{array}$ & $\begin{array}{l}\text { Área basal } \\
\left(\mathbf{m}^{2} \mathbf{h a}^{-1}\right)\end{array}$ & $\begin{array}{l}\text { Cobertura } \\
\left(\mathbf{m}^{2} \mathbf{h a}^{-1}\right)\end{array}$ & $\begin{array}{l}\text { Frecuencia } \\
\qquad(\%)\end{array}$ & $\begin{array}{l}\text { DR } \\
(\%)\end{array}$ & $\begin{array}{l}\text { FR } \\
(\%)\end{array}$ & $\begin{array}{l}\text { ABR } \\
(\%)\end{array}$ & $\begin{array}{l}\text { VIR } \\
(\%)\end{array}$ \\
\hline Alnus acuminata Kunth & 109 & 11.74 & 2013 & 30.43 & 4.84 & 3.83 & 13.44 & 22.11 \\
\hline Daphnopsis salicifolia (Kunth) Meisn. & 230 & 5.78 & 2385 & 39.13 & 10.27 & 4.92 & 6.62 & 21.81 \\
\hline Randia aff. canescens Greenm. & 309 & 1.85 & 1956 & 34.78 & 13.76 & 4.37 & 2.12 & 20.25 \\
\hline Lysiloma acapulcense (Kunth) Benth. & 61 & 7.80 & 1103 & 43.48 & 2.71 & 5.46 & 8.93 & 17.11 \\
\hline Astianthus viminalis (Kunth) Baill. & 74 & 6.67 & 963 & 30.43 & 3.29 & 3.83 & 7.64 & 14.76 \\
\hline Solanum aligerum Schltdl. & 226 & 0.55 & 1591 & 30.43 & 10.08 & 3.83 & 0.64 & 14.54 \\
\hline Salix humboldtiana Willd. & 52 & 5.40 & 1079 & 30.43 & 2.33 & 3.83 & 6.18 & 12.33 \\
\hline Sapium macrocarpum Müll.Arg. & 52 & 3.64 & 1027 & 39.13 & 2.33 & 4.92 & 4.17 & 11.41 \\
\hline Mangifera indica L. & 4 & 8.61 & 266 & 4.35 & 0.19 & 0.55 & 9.85 & 10.59 \\
\hline $\begin{array}{l}\text { Critonia quadrangularis (DC.) } \\
\text { R.M.King et H.Rob. }\end{array}$ & 78 & 1.16 & 713 & 26.09 & 3.49 & 3.28 & 1.33 & 8.10 \\
\hline Trema micrantha (L.) Blume & 74 & 2.97 & 1667 & 8.70 & 3.29 & 1.09 & 3.40 & 7.79 \\
\hline Salix taxifolia Kunth & 61 & 3.41 & 318 & 8.70 & 2.71 & 1.09 & 3.90 & 7.71 \\
\hline Psidium guajava L. & 78 & 0.80 & 596 & 26.09 & 3.49 & 3.28 & 0.91 & 7.68 \\
\hline Ficus insipida Willd. & 9 & 5.17 & 425 & 4.35 & 0.39 & 0.55 & 5.92 & 6.86 \\
\hline Inga vera Kunth & 43 & 2.21 & 665 & 17.39 & 1.94 & 2.19 & 2.53 & 6.66 \\
\hline Heliocarpus terebinthinaceus (DC.) Hochr. & 26 & 2.47 & 96 & 13.04 & 1.16 & 1.64 & 2.83 & 5.63 \\
\hline $\begin{array}{l}\text { Coursetia caribaea (Jacq.) } \\
\text { Lavin spp. caribaea }\end{array}$ & 30 & 1.77 & 505 & 13.04 & 1.36 & 1.64 & 2.03 & 5.03 \\
\hline Sassafridium macrophyllum Rose & 74 & 0.45 & 798 & 8.70 & 3.29 & 1.09 & 0.51 & 4.90 \\
\hline Alnus jorullensis Kunth & 17 & 2.43 & 423 & 8.70 & 0.78 & 1.09 & 2.78 & 4.64 \\
\hline Cordia morelosana Standl. & 35 & 0.63 & 240 & 17.39 & 1.55 & 2.19 & 0.72 & 4.46 \\
\hline Lycianthes heteroclita (Sendtn.) Bitter & 78 & 0.11 & 538 & 4.35 & 3.49 & 0.55 & 0.12 & 4.16 \\
\hline Fraxinus uhdei (Wenz.) Lingelsh. & 17 & 0.94 & 287 & 17.39 & 0.78 & 2.19 & 1.07 & 4.04 \\
\hline Solanum pseudocapsicum L. & 26 & 0.06 & 167 & 21.74 & 1.16 & 2.73 & 0.07 & 3.96 \\
\hline Critoniopsis sp. & 26 & 0.35 & 254 & 17.39 & 1.16 & 2.19 & 0.41 & 3.75 \\
\hline Aphananthe monoica (Hemsl.) J.-F.Leroy & 30 & 0.09 & 264 & 13.04 & 1.36 & 1.64 & 0.10 & 3.10 \\
\hline Annona cherimola Mill. & 22 & 0.16 & 158 & 13.04 & 0.97 & 1.64 & 0.19 & 2.80 \\
\hline $\begin{array}{l}\text { Zanthoxylum limoncello Planch. } \\
\text { et Oerst. ex Triana et Planch. }\end{array}$ & 26 & 0.43 & 336 & 8.70 & 1.16 & 1.09 & 0.50 & 2.75 \\
\hline Zinowiewia concinna Lundell & 9 & 1.53 & 201 & 4.35 & 0.39 & 0.55 & 1.75 & 2.69 \\
\hline Persea americana Mill. & 13 & 0.86 & 165 & 8.70 & 0.58 & 1.09 & 0.98 & 2.66 \\
\hline Stemmadenia obovata K.Schum. & 17 & 0.06 & 192 & 13.04 & 0.78 & 1.64 & 0.07 & 2.49 \\
\hline Acacia farnesiana (L.) Willd. var. farnesiana & רa 13 & 0.07 & 118 & 13.04 & 0.58 & 1.64 & 0.08 & 2.30 \\
\hline Ficus pertusa L.f. & 4 & 1.34 & 92 & 4.35 & 0.19 & 0.55 & 1.54 & 2.28 \\
\hline Erythrina coralloides DC. & 9 & 1.16 & 64 & 4.35 & 0.39 & 0.55 & 1.33 & 2.26 \\
\hline Diospyros digyna Jacq. & 4 & 1.05 & 87 & 4.35 & 0.19 & 0.55 & 1.20 & 1.94 \\
\hline Trichilia havanensis Jacq. & 9 & 0.32 & 139 & 8.70 & 0.39 & 1.09 & 0.36 & 1.84 \\
\hline Lysiloma sp. & 13 & 0.12 & 171 & 8.70 & 0.58 & 1.09 & 0.14 & 1.82 \\
\hline Asclepiadaceae & 13 & 0.11 & 0 & 8.70 & 0.58 & 1.09 & 0.12 & 1.80 \\
\hline Tephrosia nicaraguensis Oerst. & 13 & 0.07 & 63 & 8.70 & 0.58 & 1.09 & 0.08 & 1.76 \\
\hline Alvaradoa amorphoides Liebm. & 22 & 0.17 & 212 & 4.35 & 0.97 & 0.55 & 0.19 & 1.71 \\
\hline
\end{tabular}




\begin{tabular}{|c|c|c|c|c|c|c|c|c|}
\hline Especie & $\begin{array}{l}\text { Densidad } \\
\text { (ind. ha-1) }\end{array}$ & $\begin{array}{l}\text { Área basal } \\
\left(\mathbf{m}^{2} \mathbf{h a}^{-1}\right)\end{array}$ & $\begin{array}{c}\text { Cobertura } \\
\left(\mathbf{m}^{2} \mathbf{h a}^{-1}\right)\end{array}$ & $\begin{array}{l}\text { Frecuencia } \\
\qquad(\%)\end{array}$ & $\begin{array}{l}\text { DR } \\
(\%)\end{array}$ & $\begin{array}{l}\text { FR } \\
(\%)\end{array}$ & $\begin{array}{l}\text { ABR } \\
(\%)\end{array}$ & $\begin{array}{l}\text { VIR } \\
(\%)\end{array}$ \\
\hline Viburnum stellatum (Oerst.) Hemsl. & 9 & 0.16 & 124 & 8.70 & 0.39 & 1.09 & 0.18 & 1.66 \\
\hline Baccharis sp. & 9 & 0.09 & 38 & 8.70 & 0.39 & 1.09 & 0.10 & 1.58 \\
\hline Piper amalago L. & 9 & 0.06 & 97 & 8.70 & 0.39 & 1.09 & 0.07 & 1.55 \\
\hline Piper leucophyllum (Miq.) C.DC. & 9 & 0.05 & 57 & 8.70 & 0.39 & 1.09 & 0.06 & 1.54 \\
\hline Bocconia arborea S.Watson & 4 & 0.69 & 85 & 4.35 & 0.19 & 0.55 & 0.79 & 1.53 \\
\hline Nissolia fruticosa Jacq. & 9 & 0.01 & 0 & 8.70 & 0.39 & 1.09 & 0.02 & 1.50 \\
\hline Coursetia madrensis Micheli & 17 & 0.05 & 186 & 4.35 & 0.78 & 0.55 & 0.05 & 1.38 \\
\hline Eugenia mirandae Merr. & 13 & 0.06 & 158 & 4.35 & 0.58 & 0.55 & 0.07 & 1.19 \\
\hline Croton fragilis Kunth & 13 & 0.02 & 109 & 4.35 & 0.58 & 0.55 & 0.02 & 1.15 \\
\hline Ardisia revoluta Kunth & 4 & 0.34 & 54 & 4.35 & 0.19 & 0.55 & 0.39 & 1.13 \\
\hline Videx mollis Kunth & 9 & 0.18 & 0 & 4.35 & 0.39 & 0.55 & 0.20 & 1.13 \\
\hline Cestrum dumetorum Schltdl. & 9 & 0.09 & 94 & 4.35 & 0.39 & 0.55 & 0.11 & 1.04 \\
\hline Zanthoxylum affine Kunth & 4 & 0.21 & 72 & 4.35 & 0.19 & 0.55 & 0.24 & 0.98 \\
\hline Cedrela salvadorensis Standl. & 9 & 0.03 & 113 & 4.35 & 0.39 & 0.55 & 0.03 & 0.96 \\
\hline Guazuma ulmifolia Lam. & 9 & 0.03 & 56 & 4.35 & 0.39 & 0.55 & 0.03 & 0.96 \\
\hline Hedyosmum mexicanum Cordem. & 9 & 0.02 & 0 & 4.35 & 0.39 & 0.55 & 0.03 & 0.96 \\
\hline Ipomoea sp. & 9 & 0.02 & 0 & 4.35 & 0.39 & 0.55 & 0.03 & 0.96 \\
\hline Taxodium mucronatum Ten. & 9 & 0.02 & 56 & 4.35 & 0.39 & 0.55 & 0.02 & 0.96 \\
\hline $\begin{array}{l}\text { Vitis tiliifolia Humb. et Bonpl. } \\
\text { ex Roem. et Schult. }\end{array}$ & 9 & 0.00 & 0 & 4.35 & 0.39 & 0.55 & 0.01 & 0.94 \\
\hline Erythrina breviflora Sessé et Moc. ex DC. & 4 & 0.13 & 89 & 4.35 & 0.19 & 0.55 & 0.15 & 0.89 \\
\hline $\begin{array}{l}\text { Ageratina adenophora (Spreng.) } \\
\text { R.M.King et H.Rob. }\end{array}$ & 4 & 0.10 & 40 & 4.35 & 0.19 & 0.55 & 0.12 & 0.86 \\
\hline Guapira macrocarpa (Miranda) Miranda & 4 & 0.10 & 81 & 4.35 & 0.19 & 0.55 & 0.11 & 0.85 \\
\hline Hippocratea celastroides Kunth & 4 & 0.09 & 0 & 4.35 & 0.19 & 0.55 & 0.11 & 0.85 \\
\hline Indigofera cuernavacana Rose & 4 & 0.04 & 61 & 4.35 & 0.19 & 0.55 & 0.04 & 0.78 \\
\hline Iresine sp. & 4 & 0.01 & 39 & 4.35 & 0.19 & 0.55 & 0.02 & 0.76 \\
\hline Hibiscus spiralis Cav. & 4 & 0.01 & 23 & 4.35 & 0.19 & 0.55 & 0.01 & 0.75 \\
\hline Menispermaceae & 4 & 0.01 & 0 & 4.35 & 0.19 & 0.55 & 0.01 & 0.75 \\
\hline Rhamnus hintonii M.C.Johnst. et L.A.Johnst & st. 4 & 0.01 & 39 & 4.35 & 0.19 & 0.55 & 0.01 & 0.75 \\
\hline Senecio argutus Kunth & 4 & 0.01 & 29 & 4.35 & 0.19 & 0.55 & 0.01 & 0.75 \\
\hline $\begin{array}{l}\text { Trichilia americana (Sessé et Moc.) } \\
\text { T.D.Penn. }\end{array}$ & 4 & 0.01 & 35 & 4.35 & 0.19 & 0.55 & 0.01 & 0.75 \\
\hline Cunila lythrifolia Benth. & 4 & 0.00 & 0 & 4.35 & 0.19 & 0.55 & 0.00 & 0.74 \\
\hline Euphorbia schlechtendalii Boiss. & 4 & 0.00 & 9 & 4.35 & 0.19 & 0.55 & 0.00 & 0.74 \\
\hline Prunus cortapico Kerber ex Koehne & 4 & 0.00 & 10 & 4.35 & 0.19 & 0.55 & 0.00 & 0.74 \\
\hline Serjania triquetra Radlk. & 4 & 0.00 & 0 & 4.35 & 0.19 & 0.55 & 0.00 & 0.74 \\
\hline Trophis mexicana (Liebm.) Bureau & 4 & 0.00 & 21 & 4.35 & 0.19 & 0.55 & 0.00 & 0.74 \\
\hline
\end{tabular}

